\section{AB0271 COMPARISON OF DOSE REDUCTION METHODS BETWEEN RAPIDLY AND GRADUALLY DE-ESCALATION IN RHEUMATOID ARTHRITIS TREATED WITH BARICITINIB OVER 15 MONTHS}

M. Yamasaki ${ }^{1}{ }^{1}$ Shin-Yokohama Arthritis and Rheumatology Clinic, Rheumatology, Yokohama, Japan

Background: However tsDMARDs and treatment strategies have improved the outcomes of rheumatoid arthritis (RA), it is unknown who can taper or stop tsDMARDs and strategies for de-escalation.

Objectives: We analyze predictors of de-escalation in RA patients treated with baricitinib over 15 months in each group who start baricitinib with $4 \mathrm{mg} /$ day and $2 \mathrm{mg} /$ day.

This study will assess and compare (1) characteristic of patients who achieve remission (REM) or low disease activity (LDA) as who can taper baricitinib and (2) two de-escalation methods, rapidly and gradually de-escalation in patients who respond first-line therapy.

Methods: Cases were recruited to SHin-yokohama Arthritis REgister (SHARE) between 2015 and $2020(n=3,961)$. Patients were diagnosed according to ACR/ EULAR 2010 classification criteria and treated with baricitinib started with 4mg/ day $(n=42)$ or $2 \mathrm{mg} /$ day $(n=108)$ over 15 months. 45 cases fulfilled EULAR definition for difficult-to-treat RA (D2T-RA). In 150 (Male25, Female125 cases, RA duration 12.5+/-5.9years) cases, Clinical Disease Activity Index (CDAI), Health Assessment Questionnaire-Disability Index (HAQ-DI), anti-CCP2 and clinical parameters were analyzed. Two de-escalation methods were compared in this study. In rapidly de-escalation methods, baricitinib were stopped in patients with stable REM/LDA with no swollen joint over 12 weeks. In gradually de-escalation methods, baricitinib were decreased to $50 \%, 42 \%, 28 \%, 14 \%$ in order with stable REM/LDA with no swollen joint over 12 weeks.

Results: (1)" Detect predictors who can achieve REM/LDA with no swollen joint as starting de-escalation baricitinib" In patients started with baricitinib $4 \mathrm{mg} / \mathrm{day}$ group, 17 patients achieved REM/LDA with no swollen joint(40.5\%), there were no differences in duration of RA, onset age of RA, biologics and/or JAK inhibitors naïve, anti-CCP2 titer and CDAI at the start baricitinib between REM/LDA and non-achieved cases. In patients started with baricitinib 2mg/day group, 59 patients achieved REM/LDA with no swollen joint (54.6\%). In 2mg/day group, biologics and/or JAK inhibitors naïve was predictor for achieving REM/LDA with no swollen joint. In 2mg/day group, D2T-RA patients was negative predictor (2)"Comparison of sustained REM and/or LDA rate between rapidly and gradually de-escalation of baricitinib in rheumatoid arthritis" In whole patients, 15 patients were tapered baricitinib with rapidly de-escalation methods and 61 patients were with gradually de-escalation. Gradually de-escalation methods showed less relapse rate compared with rapidly de-escalation after tapered baricitinib (33.3\% vs. $93.8 \%, p<0.0001)$. Particularly in $2 \mathrm{mg} /$ day group, 12 patients were tapered baricitinib with rapidly de-escalation methods and 47 patients were with gradually de-escalation. Gradually de-escalation methods showed less relapse rate compared with rapidly de-escalation after tapered baricitinib for 32.7 months (33.3\% vs. $80.9 \%, p<0.0001)$. However 2 cases in $4 \mathrm{mg} /$ day group and 8 cases in $2 \mathrm{mg} /$ day showed increase of CDAl, all these cases regain LDA after increasing baricitinib.

Conclusion: Tapering baricitinib using gradually de-escalation methods may help to sucseed deduction of baricitinib in RA patients with sustained clinical REM and/or LDA with no swollen joint in each group who start baricitinib with $4 \mathrm{mg} /$ day and $2 \mathrm{mg} /$ day.

REFERENCES:

[1] Ann Rheum Dis 2019;78:171-178

Disclosure of Interests: None declared

DOI: 10.1136/annrheumdis-2021-eular.2874

\section{AB0272 1 CAN NONSTEROIDAL ANTI-INFLAMMATORY DRUGS CONTROL THE SYMPTOMS OF MODERATE RHEUMATOID ARTHRITIS?}

E. Pogozheva ${ }^{1}$, A. Karateev ${ }^{1}$, V. Amirdzhanova ${ }^{1}$, E. Filatova ${ }^{1} .{ }^{1}$ V. A. Nasonova Research Institute, Pain Management, Moscow, Russian Federation

Objectives: to evaluate the efficacy of long-term pain therapy with nonsteroidal anti-inflammatory drugs (NSAIDs) in patients with rheumatoid arthritis (RA) with an initially moderate disease activity (DAS $28<5,1$ ).

Methods: the study included 404 RA patients, disease duration was more than 1 year, mean DAS $283.7 \pm 1.6$, mean age $58.6 \pm 10.0$ years, $69 \%$ women, $76,7 \%$ RF "+," $81,5 \%$ ACPA "+". $91,2 \%$ of the patients received conventional DMARDs (methotrexate), $8,8 \%$ - biological agents. All patients received NSAIDs (aceclofenac) to control their symptoms. The follow-up period was 6 months. We evaluated the dynamics of the DAS 28 index, the level of pain and patient global health on a 100- $\mathrm{mm}$ visual analog scale (VAS).

Results: the level of pain (VAS) decreased from $63,1 \pm 15,4$ to $46,3 \pm 8,3$ $(p=0,001)$ by 3 months of follow-up and up to $39,5 \pm 11,2(p=0,001)$ by 6 months of follow-up. The patient global health (VAS) also improved from 58,2 $\pm 13,4$ at baseline to $40,3 \pm 11,2(p=0,001)$ at 3 months and to $35,5 \pm 9,7(p=0,001)$ at 6 months of follow up. The mean DAS 28 remained within the moderate disease activity and decreased from $3,7 \pm 1,5$ to $3,4 \pm 1,1(p=0,01)$ after 3 months, and to $3,1 \pm 0,9(p=0,01)$ after 6 months.

Conclusion: long-term NSAID therapy allows to control the disease activity in patients with moderate RA. This should be taken into account when planning therapy, including deciding whether to "switch" DMARDs and prescribing biological agents.

Disclosure of Interests: None declared

DOI: 10.1136/annrheumdis-2021-eular.2901

\section{AB0273 HYPERSENSIVITY REACTIONS TO NON STEROID ANT INFLAMMATORY DRUGS: A BOUT 87 CASES}

K. Ksouda ${ }^{1}$, R. Sahnoun ${ }^{1}$, R. Atheymen ${ }^{1}$, I. Bouaziz ${ }^{1}$, A. Hanène ${ }^{1}$,

S. Hammami ${ }^{1}$, L. Chtourou ${ }^{2}$, Z. Khaled ${ }^{1} .{ }^{1}$ Medecine School of Sfax,

Pharmacovigilance Department, Sfax, Tunisia; ${ }^{2}$ Hedi Chaker Hopital,

Gastroenterology Department, Sfax, Tunisia

Background: Non-steroidal anti-inflammatory drugs (NSAIDs) are one of the leading causes of hypersensitivity reactions to drugs. The pathogenesis may be immunological mechanisms (allergic reactions) or non specific immunological reactions often incriminated in cross reactivity independently of chemical structure of these molecules. Understanding of the underlying mechanism is necessary for prevention and choice of safe alternatives [1, 2]

Objectives: Analyze all cases of non-steroidal anti-inflammatory drugs cutaneous eruption reported to sfax pharmacovigilance service since January 2015 to December 2020 and evaluate the possibility of cross-reactions between different molecules in this class.

Methods: We conducted a retrospective study of all cases reported to sfax phar macovigilance department. An enquiry of pharmacovigilance was performed in patients who presented side effects to AINS. The imputability study was carried out by the French method of Imputability. Medical history specifies if there is a re-administration to assess tolerance and cross-reactivity.

Results: Our study included 87 patients whose average age was 45,8 years The sex ratio $(\mathrm{F} / \mathrm{M})$ was 1.18 . Iysine salicylate acetyl is the most incriminated (31\%), then mefenamic acid (19.5\%), diclofenac (19.5\%), ketoprofen in $(9.2 \%)$ piroxicam in $(6.9 \%)$, ibuprofen in $(5.4 \%)$, celocoxib in $(3.4 \%)$, tiaprofenic acid in $(1.1 \%)$ and naproxen in $1.1 \%$ of cases. The most common skin injury was urticaria in 29 cases (33.3\%). Fixed drug eruption was observed in 17 cases. Maculopapular rash was observed in 19 cases, anaphylaxis in 5 cases and 4 cases of photosensitivity were observed. In our study we found cross-reactivity between (NSAIDs) in 8 patients.

Conclusion: The diagnostic approach is often based on the controlled administration of the drug to assess tolerance and to identify safe alternatives. In cases of intolerance to COX 1 inhibitors, cross-reactions to selective cox 2 inhibitors are very rare [3]

\section{REFERENCES:}

[1] Inmaculada Dona, Maria Salas, James R Perkins and al. Hypersensitivity Reactions to Non-Steroidal Anti-Inflammatory Drugs. Curr Pharm Des 2016; 22(45):6784-6802.

[2] Flavia Angeletti, Franziska Meier, Nadja Zöller, Markus Meissner, Roland Kaufmann, Eva Maria Valesky. Hypersensitivity reactions to non-steroida anti-inflammatory drugs (NSAIDs) - a retrospective study. J Dtsch Dermato Ges 2020 Dec; 18(12):1405-1414.

[3] N Blanca-López, J A Cornejo-García, M C Plaza-Serón, and al. Hypersensitivity to Nonsteroidal Anti-inflammatory Drugs in Children and Adolescents: Cross-Intolerance Reactions. J Investig Allergol Clin Immunol 2015; 25(4):259-69.

Disclosure of Interests: None declared

DOI: 10.1136/annrheumdis-2021-eular.2910

\section{AB0274 METHOTREXATE INTOLERANCE IN MOROCCAN RHEUMATOID ARTHRITIS PATIENTS}

M. Mahroug ${ }^{1}$, H. Azzouzi ${ }^{1}$, H. Boutaibi ${ }^{1}$, O. Lamkhanat ${ }^{1}$, I. Linda ${ }^{1} .{ }^{1}$ Mohammed VI University Hospital, Mohammed I University, Faculty of Medicine, Rheumatology, Oujda, Morocco

Background: Methotrexate intolerance is a principal reason for treatment discontinuation, hence the interest in a more in-depth study.

Objectives: We aimed to study the prevalence of methotrexate gastrointestinal intolerance and determine its associated factors in rheumatoid arthritis (RA) patients.

Methods: We designed a cross-sectional study on our RA patients recruited in January 2021 at our rheumatology department. Methotrexate Intolerance Severity Score (MISS) [1], previously validated in juvenile idiopathic arthritis 
patients, was used to determine methotrexate (MTX) intolerance prevalence in RA patients. The MISS consisted of four domains: abdominal pain, nausea, vomiting, and behavioral symptoms, occurring before (anticipatory), after, and when thinking of MTX (associative). MTX intolerance was defined as six or more points on the MISS. Our statistical analysis was based on a descriptive study and logistic regression with SPSS20.

Results: We included 102 RA patients with a mean age of $51.60 \pm 14.33$ years, Women were predominant $(93.1 \%)$. The mean disease duration was $14.86 \pm$ 9.78 years, with a mean methotrexate use duration of $7.42 \pm 6.44$ years. The mean dose of methotrexate was $12.13 \pm 9.06 \mathrm{mg}$ per week. The prevalence of methotrexate intolerance was $55.9 \%$, and seventy-six patients $(74.5 \%)$ experienced at least one gastrointestinal symptom during MTX treatment. After MTX administration, the most prevalent gastrointestinal symptom was nausea $(93 \%$ of the intolerant patient), whereas abdominal pain occurred in $73.7 \%$ and vomiting in $57.9 \%$. These symptoms were also prevalent before and when thinking of MTX. Anticipatory nausea was reported in $45.6 \%$ and associative nausea in $54.5 \%$ of the cases, abdominal pain occurred anticipatory in $22.8 \%$ and associative in $42.1 \%$, anticipatory vomiting was the least prevalent, affecting $8.8 \%$. Behavioral symptoms affected $87.7 \%$ of intolerant patients, with restlessness being the most prominent symptom in $71.9 \%$ of them. Among the intolerant patients, 45 patients (79\%) took parenteral MTX, and $12(21.1 \%)$ took methotrexate orally. In comparison, young patients $(49.11 \pm 14.95$ years) were more intolerant to MTX than old $(54.76 \pm 13$ years, $p=0.048)$ ones. However, in univariate logistic regression analysis, we did not find any significant association between methotrexate administration route, dose, duration, and digestive intolerance.

Conclusion: Methotrexate intolerance was highly prevalent in our RA population. These results strengthen the idea that early detection of MTX intolerance may avoid effective treatment discontinuation, especially in younger patients.

REFERENCES:

[1] Bulatović M, Heijstek MW, Verkaaik M, van Dijkhuizen EH, Armbrust W, Hoppenreijs EP et al. High prevalence of methotrexate intolerance in juvenile idiopathic arthritis: development and validation of a methotrexate intolerance severity score. Arthritis Rheum. 2011 Jul; 63(7):2007-13.

Disclosure of Interests: None declared

DOI: 10.1136/annrheumdis-2021-eular.3315

\section{AB0275 RHEUMATOID ARTHRITIS AFTER CONVENTIONAL OR BIOLOGICAL THERAPY - IT REAL ROLE IN DIFFERENT LINES OF TREATMENT}

W. Rivero ${ }^{1}$, L. Ibata ${ }^{2}$, S. Martinez ${ }^{2}$, A. Rojas-Villarraga ${ }^{3}$, P. Santos-Moreno ${ }^{4}$ ${ }^{1}$ Biomab - Center for Rheumatoid Arthritis, Bogota, Colombia, Pharmaceutical chemist, Bogota, Colombia; ${ }^{2}$ Epithink Health Consulting, Epidemiology, Bogota, Colombia; ${ }^{3}$ Fundacion Universitaria de Ciencias de la Salud - FUCS, Research Division, Bogota, Colombia; ${ }^{4}$ Biomab - Center for Rheumatoid Arthritis, Bogota, Colombia, Rheumatology, Bogota, Colombia

Background: rheumatoid arthritis (RA) is a chronic and disabling autoimmune disease, with a high clinical and economic burden. This implies the need to investigate therapies that maximize clinical results. Tofacitinib is recommended as a different alternative to biologic therapy when a patient remains with moderate or high disease activity after conventional DMARDs use, or as an option after failure to biologic therapy.

Objectives: to evaluate the effectiveness of Tofacitinib in RA as first-line (after conventional DMARDs failure) or second-line treatment (after biologic therapy failure) in a real-life cohort of RA patients and its differences.

Methods: this is a descriptive retrospective cohort study conducted at a specialized center for RA in Bogota, Colombia; databases from 2017 to 2019 were used to select and study patients with indication of Tofacitinib, regardless of their previous treatment or disease status. The indication and initiation of Tofacitinib (5 mg BID or $11 \mathrm{mg}$ once daily) was an independent medical decision made as part of the individualized management of every patient. Effectiveness was evaluated in those patients who met the high adherence criteria (at least three visits with a rheumatologist per year), with no change or addition of other conventional DMARDs. Frequencies and proportions in baseline characteristics, differences in disease activity were calculated between the first and second line tofacitinib treatment. Comparisons of continuous variables data between the two patient groups were made using the t-test; the chi-square test and Fisher's exact test were used for statistical analysis of categorical variables. Logistic regressions were performed to analyze related factors with therapeutic response outcomes. Results: we included 152 RA patients who received tofacitinib: first-line (T1) ( $n=$ $85,55.9 \%)$ or second-line (T2) ( $n=67,44.1 \%)$. T1 first-line group was younger than the T2 second-line patients $(53 \pm 12.8$ years and $59 \pm 11.4$ years, p-value 0.01$)$ and they had a shorter disease duration than T2 patients (11.8 vs. 12.8 years, $\mathrm{p}$-value 0.01$)$. Comparative analysis of response to treatment showed a reduction in DAS28 at 3, 6 and 12 months in both study groups. The response in disease activity at 3 months was a major factor related to 6-month response (OR 13.4,
95\% Cl 4.5-39.4, p value 0.000), while non-response at 3 months were associated with no response at 6 months of follow-up. Baseline DAS28 was significantly associated with response at 12 months (OR 1.9, 95\% Cl 1.11-3.25, p-value 0.028). At 12 months of treatment, both groups showed disease response and control according to the DAS28 from baseline, but a higher proportion of T1 patients achieved remission ( $45 \%$ vs $23 \%$ ). A subgroup analysis to evaluate T2 second-line Tofacitinib therapy showed no statistically significant differences in any response criteria according to the number of previously received biologicals.

Table 1. Regression analysis (risk of response of the disease at 6 and 12 months of treatment with Tofacitinib)

\begin{tabular}{lcccccc}
\hline \multirow{2}{*}{ Factor } & \multicolumn{3}{c}{ Response at Month 6 } & \multicolumn{3}{c}{ Response at Month $12^{*}$} \\
\cline { 2 - 7 } & OR & IC95\% & P value & OR & IC95\% & P value \\
\hline Age & 1.00 & $0.97-1.03$ & 0.788 & 1.02 & $0.98-1.06$ & 0.211 \\
Male & 1.82 & $0.65-5.08$ & 0.251 & 0.81 & $0.27-2.38$ & 0.709 \\
Duration of RA & 0.99 & $0.94-1.04$ & 0.908 & 1.02 & $0.96-1.08$ & 0.444 \\
Positive Rheumatoid Factor & 0.81 & $026-2.56$ & 0.730 & 0.63 & $0.17-2.26$ & 0.485 \\
Positive Anti-CCP & 0.34 & $0.068-1.6$ & 0.189 & & & \\
Initial DAS28 & 1.61 & $1.04-2.49$ & 0.033 & 1.9 & $1.11-3.25$ & 0.018 \\
First line & 0.44 & $0.19-1.01$ & 0.054 & 1.47 & $0.56-3.83$ & 0.423 \\
Treatment period & 1.12 & $0.80-1.55$ & 0.492 & 1.1 & $0.75-1.61$ & 0.607 \\
Dose: 11 mg. & 0.95 & $0.42-2.13$ & 0.904 & 0.75 & $0.258-1.9$ & 0.565 \\
Response at Month 3 & 13.42 & $4.57-39.4$ & 0.000 & 2.32 & $0.87-6.18$ & 0.091 \\
\hline
\end{tabular}

${ }^{*}$ Positive Anti-CCP at month 12 was omitted because of collinearity

Conclusion: Tofacitinib is an effective treatment option for patients with RA after conventional DMARDs and in patients after biologic therapy failure, but maybe is better used it as a T1 first-line of treatment. Further studies are required to determine the real role of tofacitinib in different lines of RA treatment.

Disclosure of Interests: Wilberto Rivero: None declared, Linda Ibata: None declared, Susan Martinez: None declared, Adriana Rojas-Villarraga: None declared, Pedro Santos-Moreno Speakers bureau: Pedro Santos-Moreno has received fees for conferences from: Abbvie, Biopas-UCB, Bristol, Janssen, Pfizer Sanofi., Consultant of: Pedro Santos-Moreno has received fees for counseling and advisory boards from: Abbvie, Abbott, Biopas-UCB, Bristol, Janssen, Pfizer, Roche, Sanofi., Grant/research support from: Pedro Santos-Moreno has received research grants from: Abbvie, Abbott, Biopas-UCB, Bristol, Janssen, Pfizer, Roche, Sanofi. DOI: 10.1136/annrheumdis-2021-eular.3770

\section{AB0276 HOW FAST CAN METHOTREXATE BE ESCALATED IN RHEUMATOID ARTHRITIS? A MULTICENTRE, PARALLEL-GROUP RANDOMIZED CONTROLLED TRIAL (MEIRA)}

S. Jain ${ }^{1}$, V. Dhir ${ }^{1}$, A. Aggarwal ${ }^{2}$, S. Maurya ${ }^{2}$, R. Gupta ${ }^{3}$, B. Leishangthem ${ }^{1}$, A. Khullar ${ }^{1}$, V. Dhawan ${ }^{4}$, G. Naidu' ${ }^{1}$, S. K. Sharma ${ }^{1}$, A. Sharma ${ }^{1}$, S. Jain ${ }^{1}$. ${ }^{1}$ PGIMER, Division of Clinical Immunology and Rheumatology, Department of Internal Medicine, Chandigarh, India; ${ }^{2}$ SGPGIMS, Department of Clinical Immunology and Rheumatology, Lucknow, India; ${ }^{3}$ AIIMS, Department of Rheumatology, New Delhi, India; ${ }^{4}$ PGIMER, Department of Experimental Medicine and Biotechnology, Chandigarh, India

Background: Literature regarding the optimal dose escalation strategy of methotrexate (MTX) in RA is scant and ambiguous (1). Concerns regarding the safety of rapid escalation may lead to delayed attainment of the optimal dose and treatment target.

Objectives: To compare the efficacy, safety and tolerability of fast versus usual dose escalation of oral MTX in RA.

Methods: This multicenter, open-label (assessor blinded) RCT included patients with active $R A$ ( $S J C \geq 2$ and $T J C \geq 4$ ) aged $18-55$ years, not on DMARDs (excep HCQ and/or low-dose prednisolone) and with disease duration $<5$ years. Patients were randomized 1:1 into two groups with the same starting dose of oral MTX ( $15 \mathrm{mg} /$ week), but escalated either by $5 \mathrm{mg}$ every 2 weeks (fast escalation group) or $5 \mathrm{mg}$ every 4 weeks (usual escalation group), till a maximum of $25 \mathrm{mg} / \mathrm{wk}$ Primary outcome was proportion of EULAR good responders at 16 weeks. Secondary outcomes were change in DAS28-3 and EULAR responders (good or moderate) at 8 and 16 weeks, change in Indian $\mathrm{HAQ}$ at 16 weeks, and symptomatic (questionnaire based) and laboratory adverse effects over 16 weeks. RBC MTX polyglutamate-3 levels were measured using HPLC in both groups. There was an open-label extension phase till 24 weeks (use of other DMARDs was permitted beyond 16 weeks if target was not met), and DAS28-3 at 24 weeks was compared. Trial Reg: CTRI/2018/12/016549

Results: 178 patients (mean age 39.8 (8.6) years, $84 \%$ females) with mean disease duration of 1.9 (1.4) years were randomized (89 in each group). Mean DAS28ESR-3 and DAS28CRP-3 at enrollment were $6.3(0.9)$ and 5.4 (1.1). At 16 weeks, there was no significant difference in good EULAR response by DAS28ESR-3 (5.6, 7.9\%, $\mathrm{p}=0.9$ ) or DAS28CRP-3 $(28.1,22.5 \%, \mathrm{p}=0.8)$ between the two groups (Figure 1). 